SUPPORTING INFORMATION FOR:

\title{
Spectroscopic Study of Sol-Gel Entrapped Triruthenium Dodecacarbonyl Catalyst Reveals Hydride Formation
}

Joel G. Patrow; Yukun Cheng; Cynthia G. Pyles; Bing Luo; Ian A. Tonks; Aaron M. Massari*

University of Minnesota - Twin Cities, 207 Pleasant St. SE, Minneapolis, MN 55454

Corresponding author: massari@umn.edu 


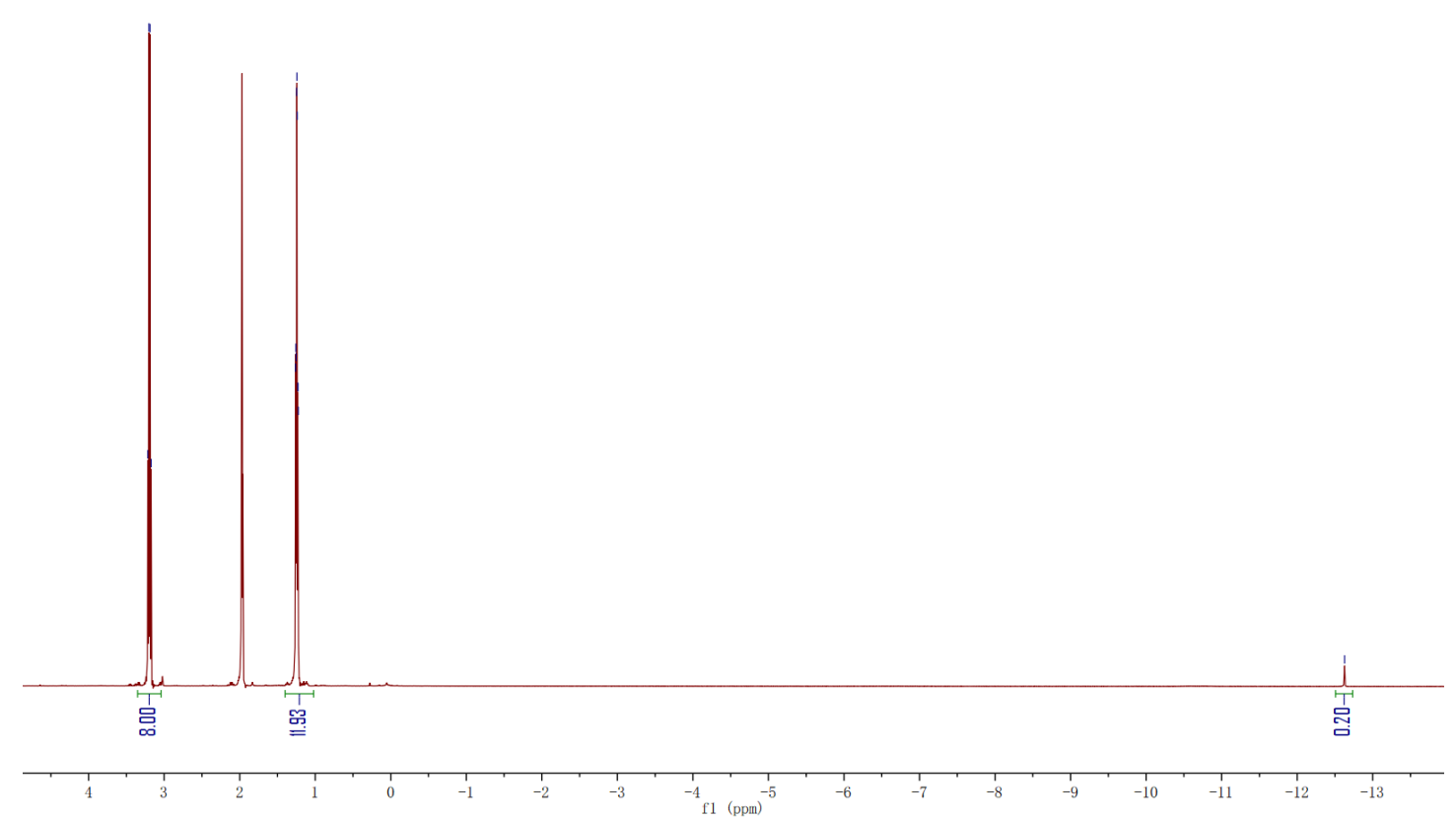

Figure S1. ${ }^{1} \mathrm{HNMR}$ spectrum of $\left[\mathrm{NEt}_{4}\right]\left[\mathrm{HRu}_{3}(\mathrm{CO})_{11}\right]$. 

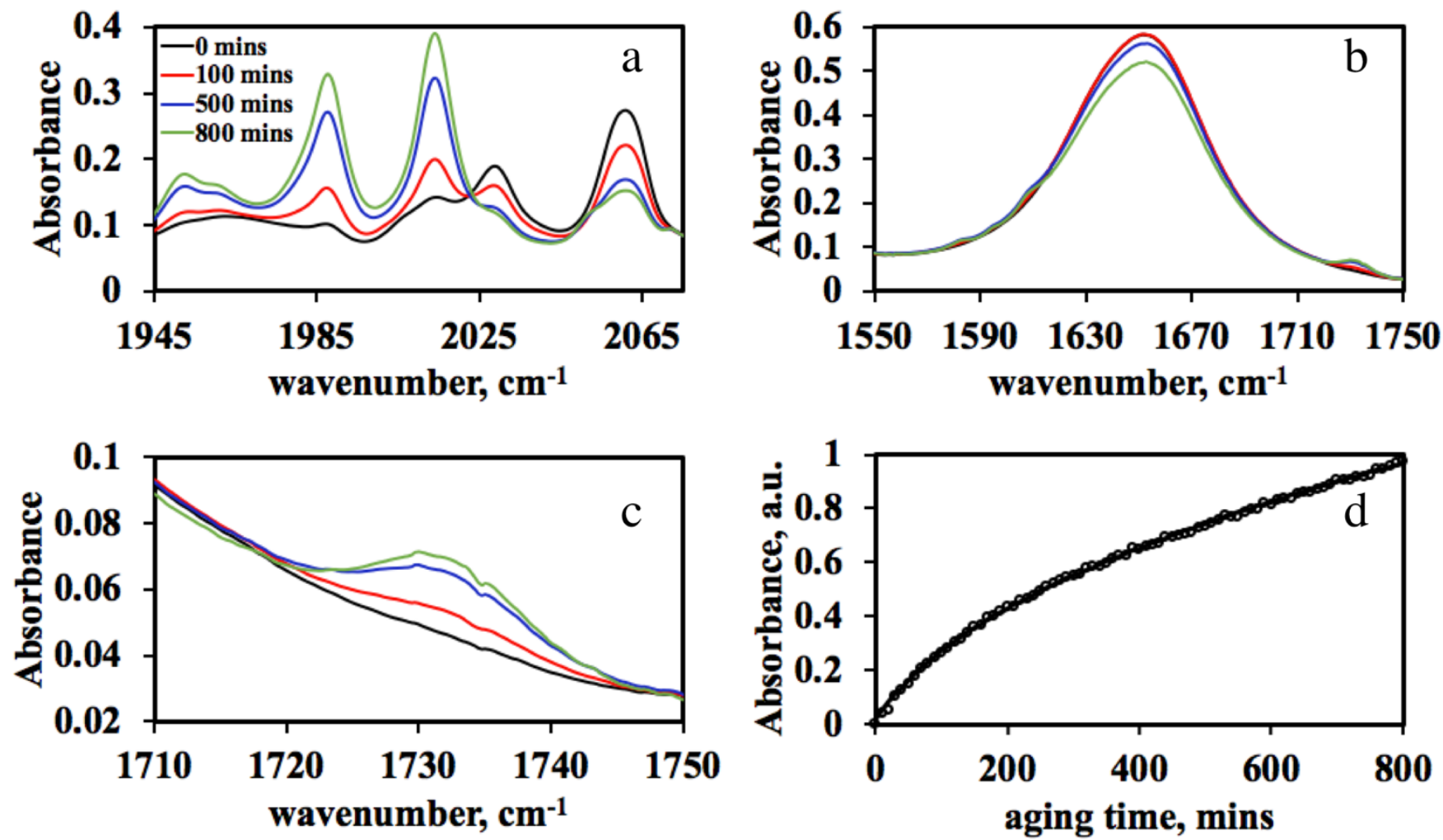

Figure S2. (a-c) FTIR spectra of the $\mathrm{Ru}_{3}(\mathrm{CO})_{12}$ during the sol-gel aging process. a) Spectra of the carbonyl modes. b) Spectra of the bridging carbonyl at $1733 \mathrm{~cm}^{-1}$ and water bend at $1650 \mathrm{~cm}^{-1}$. c) Zoomed in spectra of the bridging carbonyl mode. d) Plot of the absorbance of the bridging carbonyl as a function of aging time (note: because the bridging mode overlaps with the wing of the water bend peak, it was necessary to divide out the kinetics of the water peak). d) Kinetics of the bridging carbonyl peak with the water dynamics divided out. The solid line is the basis spectrum fit for coefficient B. The dynamics of $\mathrm{B}$ and the bridging carbonyl are the same. 


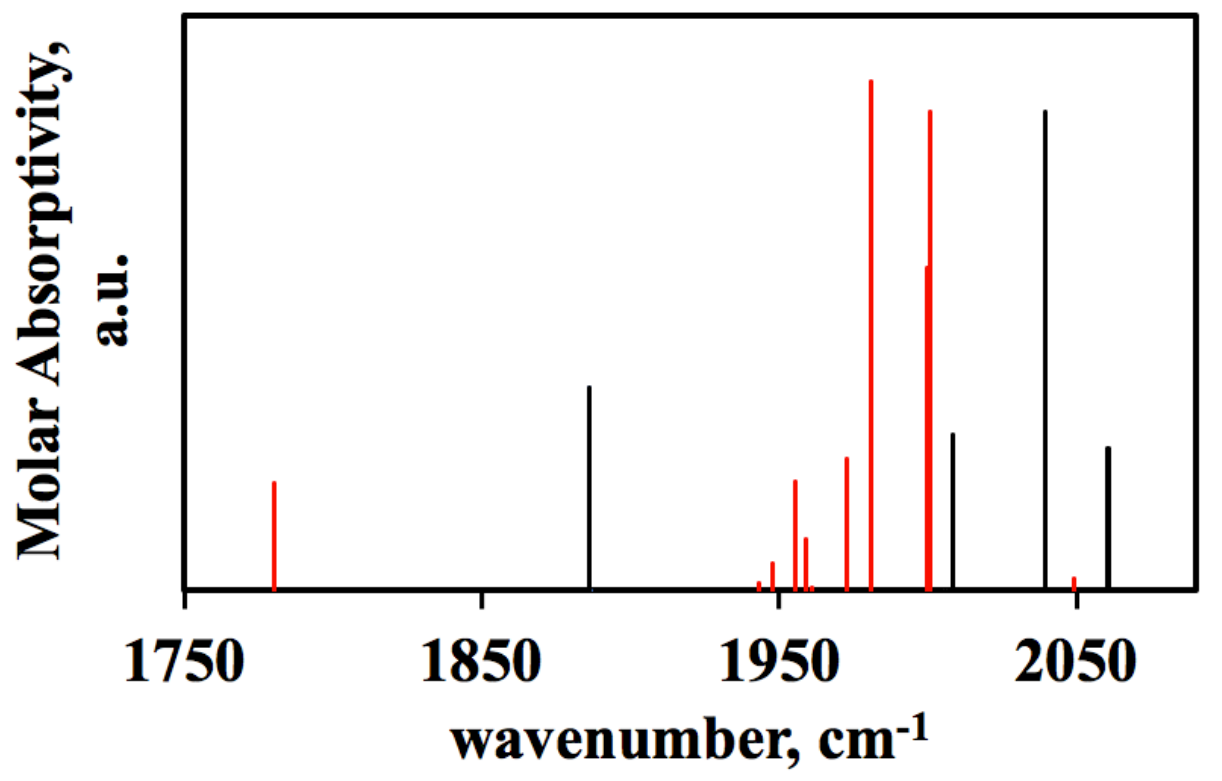

Figure S3. Computational vibrational frequencies (relevant to this work) and their computed molar absorptivities. The black lines are for $\mathrm{Ru}_{3}(\mathrm{CO})_{12}$ while the red ones are for $\left[\mathrm{HRu}_{3}(\mathrm{CO})_{11}\right]^{-}$. The red line at $1780 \mathrm{~cm}^{-1}$ corresponds to the bridging carbonyl stretch.

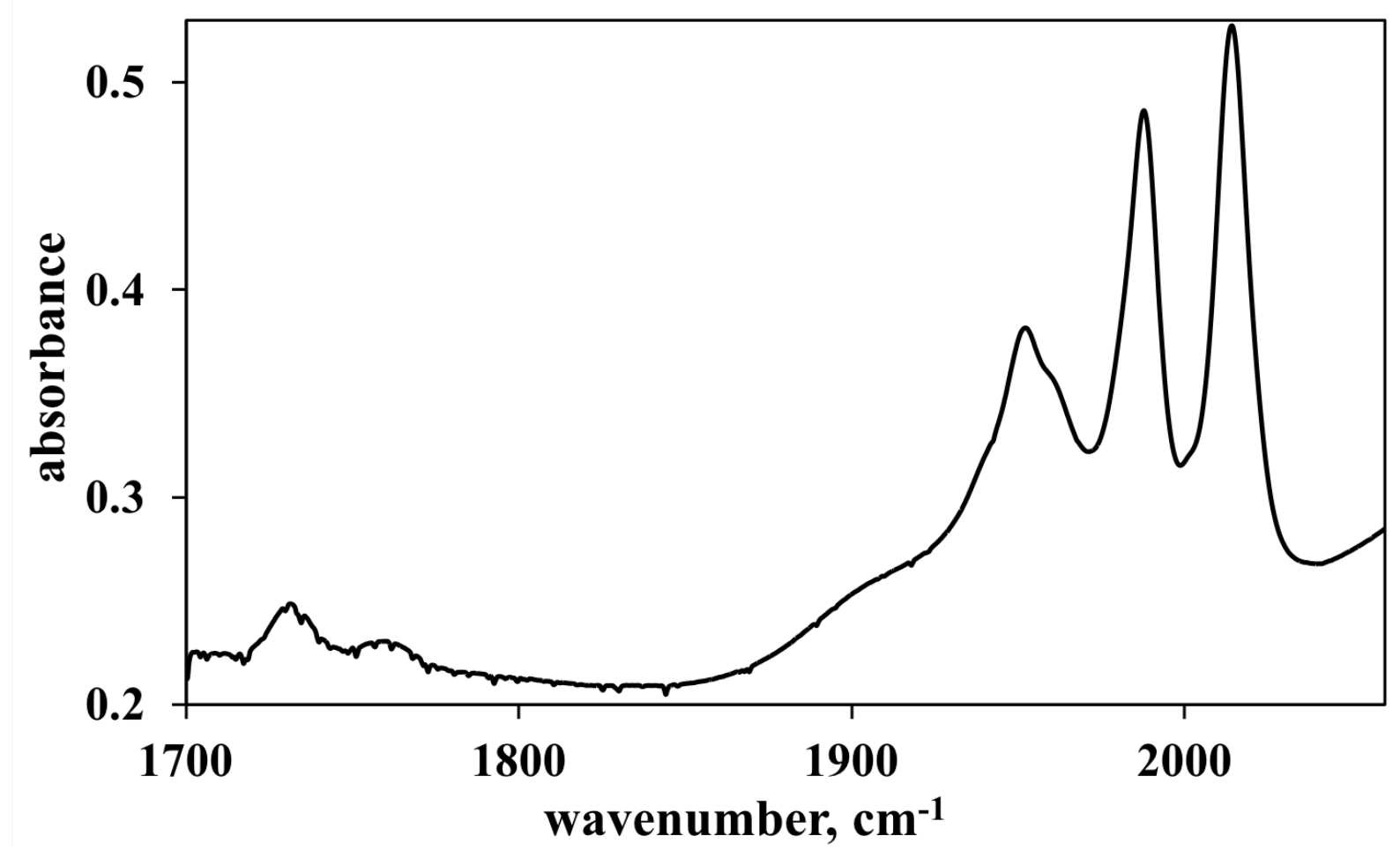

Figure S4: FTIR spectrum of $\mathrm{D}_{2} \mathrm{O}$ sol-gel encapsulation of $\mathrm{Ru}_{3}(\mathrm{CO})_{12}$, i.e. $\left[\mathrm{HRu}_{3}(\mathrm{CO})_{11}\right]$ - 


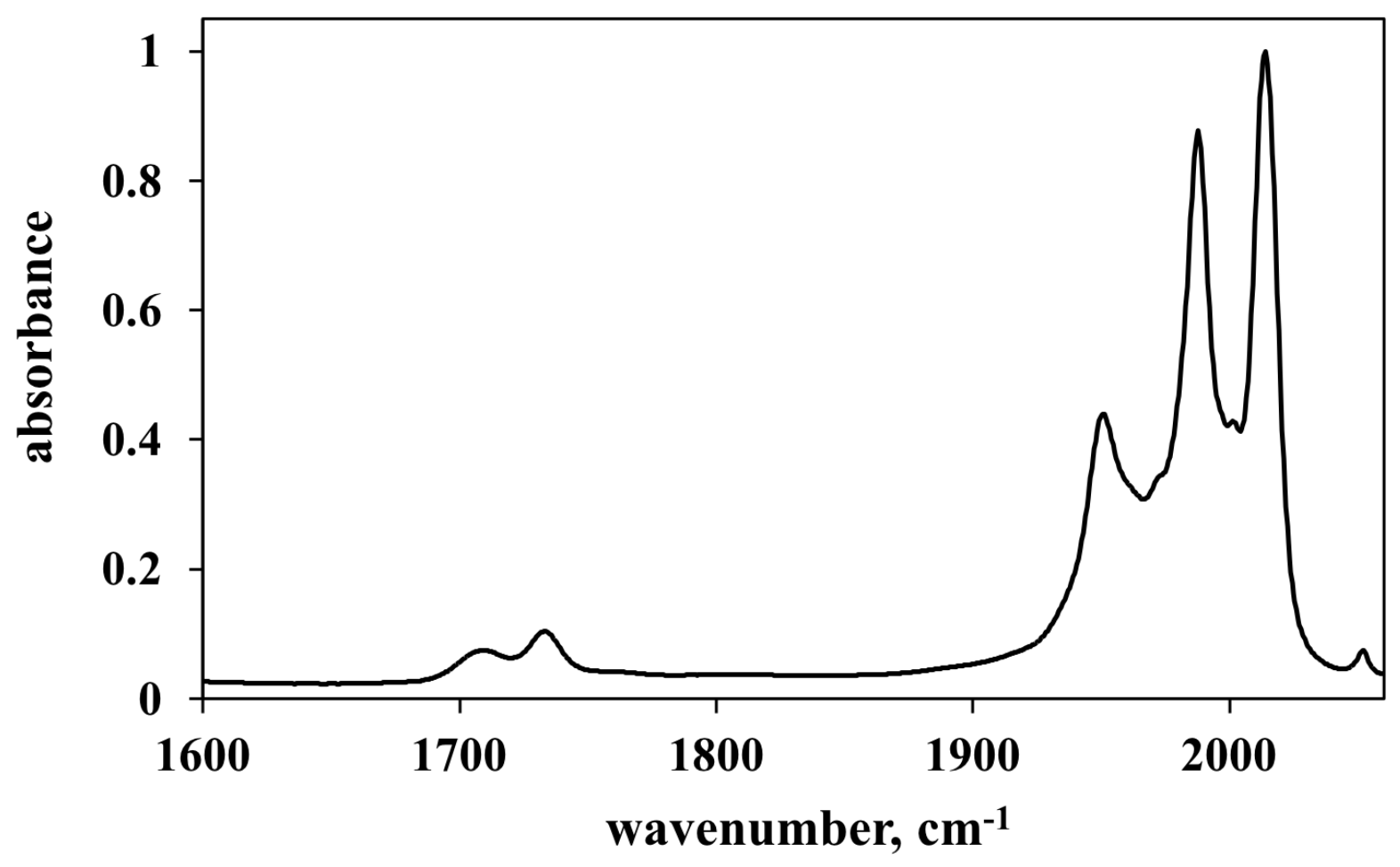

Figure S5: FTIR spectrum of $\left[\mathrm{NEt}_{4}\right]\left[\mathrm{DRu}_{3}(\mathrm{CO})_{11}\right]$ in THF. 
Table S1. Complete list of computational frequencies and their normal modes.

\begin{tabular}{|c|c|c|c|}
\hline \multicolumn{2}{|c|}{$\mathrm{Ru}_{3}(\mathrm{CO})_{12}$} & \multicolumn{2}{|c|}{$\left[\mathrm{HRu}_{3}(\mathrm{CO})_{11}\right]^{-}$} \\
\hline wavenumber $\left(\mathrm{cm}^{-1}\right)$ & Int. $\left(\mathrm{M}^{-1} \mathrm{~cm}^{-1}\right)$ & Wavenumber $\left(\mathrm{cm}^{-1}\right)$ & Int. $\left(\mathrm{M}^{-1} \mathrm{~cm}^{-1}\right)$ \\
\hline 34.640736 & 0.05527916 & 32.046912 & 0.01195067 \\
\hline 49.649664 & 0.00771369 & 33.070848 & 10.2025589 \\
\hline 51.330336 & 0.09699465 & 36.7272 & 0.64652065 \\
\hline 51.443904 & 0.32012019 & 43.944864 & 0.14815603 \\
\hline 51.51456 & 1.51662673 & 47.24112 & 17.5840091 \\
\hline 51.61584 & 1.40977282 & 59.291136 & 9.20457256 \\
\hline 57.021408 & 0.52388408 & 65.488512 & 0.02339233 \\
\hline 62.682144 & 9.01822461 & 67.530432 & 1.95091096 \\
\hline 71.04912 & 0 & 68.987808 & 1.89304338 \\
\hline 71.31792 & 0 & 70.524096 & 17.5568545 \\
\hline 79.541184 & 1.04964629 & 71.255904 & 6.25082576 \\
\hline 79.920288 & 0.99674675 & 75.539712 & 3.11801589 \\
\hline 82.081152 & 57.6751452 & 81.011328 & 3.43217483 \\
\hline 83.823936 & 1.10110194 & 81.742752 & 7.87584194 \\
\hline 83.972544 & 1.1173965 & 81.809472 & 0.40259965 \\
\hline 87.589632 & 0.00874492 & 84.3456 & 0.04994693 \\
\hline 87.799776 & 0.00872399 & 87.418656 & 10.4530938 \\
\hline 100.444992 & 0 & 91.54272 & 77.6986361 \\
\hline 100.490208 & 0 & 100.150656 & 8.7609222 \\
\hline 105.565344 & 0.00362792 & 105.105984 & 16.3969701 \\
\hline 109.943424 & 1.74172377 & 119.3784 & 0.91111064 \\
\hline 116.482176 & 0.02959113 & 136.133472 & 2.76827168 \\
\hline 123.066432 & 2.11615676 & 137.963712 & 93.450016 \\
\hline 123.909408 & 2.33975363 & 180.375552 & 0.75587653 \\
\hline 154.454304 & 0.00743875 & 208.70208 & 124.802858 \\
\hline 179.093952 & 229.253886 & 326.694624 & 78.5835565 \\
\hline 179.38272 & 230.163701 & 332.786496 & 2.92196856 \\
\hline 281.085888 & 636.977208 & 348.540192 & 17.755796 \\
\hline 281.3448 & 644.205987 & 350.092992 & 0.39600775 \\
\hline 307.368384 & 0.02118206 & 351.575136 & 31.3901994 \\
\hline 311.035776 & 0.01600706 & 367.630752 & 3.36383547 \\
\hline 344.713248 & 0.10110251 & 370.788 & 70.5895717 \\
\hline 344.81568 & 0.05553433 & 380.960544 & 140.537842 \\
\hline 349.237344 & 1418.59646 & 384.629952 & 211.742964 \\
\hline 349.714368 & 1427.04544 & 388.582752 & 133.832873 \\
\hline 353.830176 & 0.2954924 & 399.720672 & 70.351246 \\
\hline
\end{tabular}




\begin{tabular}{|c|c|c|c|}
\hline 353.86944 & 0.04220852 & 404.816736 & 9.36791618 \\
\hline 362.061888 & 745.33252 & 414.998688 & 256.010073 \\
\hline 380.302176 & 110.591883 & 421.916832 & 133.070776 \\
\hline 388.629792 & 0.00886921 & 423.952128 & 12.4727161 \\
\hline 388.698144 & 0.01675001 & 431.53248 & 166.841625 \\
\hline 400.88016 & 3.89306435 & 431.575296 & 306.52217 \\
\hline 400.953984 & 3.67552231 & 436.10592 & 20.3256169 \\
\hline 408.947232 & 0.04214284 & 441.153216 & 59.9588628 \\
\hline 409.752096 & 3.37975929 & 450.611424 & 8.65640104 \\
\hline 434.278752 & 45.8093049 & 458.425152 & 42.5208835 \\
\hline 434.390112 & 45.9007149 & 467.672448 & 195.827845 \\
\hline 446.30064 & 0.00429063 & 468.454368 & 141.355844 \\
\hline 451.027008 & 0.0025474 & 478.921632 & 49.7014647 \\
\hline 460.971552 & 17.830124 & 490.130016 & 43.1436063 \\
\hline 461.229312 & 18.5732878 & 514.041408 & 396.26519 \\
\hline 466.357536 & 0.01970928 & 526.252128 & 380.698364 \\
\hline 466.646304 & 0.07550549 & 534.3888 & 2540.67972 \\
\hline 472.909728 & 379.597231 & 539.747328 & 87.79009 \\
\hline 531.314112 & 0.00432492 & 550.192032 & 0.01670612 \\
\hline 531.361536 & 0.00360378 & 555.728352 & 1143.26158 \\
\hline 536.36544 & 555.529125 & 561.245568 & 1551.41102 \\
\hline 565.325088 & 1258.61535 & 606.774432 & 971.923798 \\
\hline 565.390368 & 1256.08296 & 701.2824 & 274.631252 \\
\hline 572.356992 & 0.14319416 & 1140.1608 & 250.888133 \\
\hline 580.153536 & 35.930722 & 1269.96787 & 15.9574907 \\
\hline 580.346208 & 35.9550888 & 1780.07597 & 1021.01567 \\
\hline 597.041568 & 0.00641467 & 1943.11613 & 64.65054 \\
\hline 1886.00256 & 2192.16675 & 1947.53424 & 259.162758 \\
\hline 1886.2536 & 2196.46894 & 1955.4672 & 1035.02256 \\
\hline 1886.50464 & 0.08487146 & 1958.94 & 486.742797 \\
\hline 2005.72541 & 0.30207413 & 1960.91309 & 26.2210849 \\
\hline 2005.84272 & 0.40726071 & 1972.4208 & 1246.75705 \\
\hline 2008.29187 & 1683.93221 & 1980.80986 & 4847.46842 \\
\hline 2008.43491 & 1673.49841 & 1999.49578 & 3066.28243 \\
\hline 2019.24029 & 0.16614782 & 2000.72016 & 4566.49976 \\
\hline 2039.38214 & 5196.88905 & 2049.29462 & 104.740744 \\
\hline 2060.47699 & 1545.58025 & --- & --- \\
\hline 2060.57606 & 1541.4036 & $\begin{array}{l}--- \\
\end{array}$ & --- \\
\hline 2105.74819 & 0.00672936 & --- & --- \\
\hline
\end{tabular}


Table S2. Complete list of XPS binding energies.

\begin{tabular}{ccc} 
Peak assignment & $\begin{array}{c}\text { Binding Energy, eV } \\
\left(\mathrm{Ru}_{3}(\mathrm{CO})_{12}\right)\end{array}$ & $\begin{array}{c}\text { Binding Energy, eV } \\
\left(\left[\mathrm{HRu}_{3}(\mathrm{CO})_{11}\right]^{-}\right)\end{array}$ \\
\hline $3 \mathrm{~d}_{5 / 2}$ & 281.8 & 281.3 \\
$3 \mathrm{~d}_{3 / 2}$ & 286.3 & 285.8 \\
$\mathrm{C} 1 \mathrm{~s}$ (adventitious C) & 284.8 & 284.8 \\
$\mathrm{C} 1 \mathrm{~s}(\mathrm{CO})$ & 287.8 & 287.0
\end{tabular}

